\title{
Alternativas de modelos de negocios para las mipymes colombianas desde la responsabilidad social empresarial, el comercio justo, los negocios inclusivos y el valor compartido
}

Patricia Hernández-Bernal*

Candidata a doctora en Educación. Profesora investigadora, Universidad de La Salle, Bogotá, Colombia. Correo electrónico:

haphernandez@unisalle.edu.co.

Recibido: 6 de septiembre del 2015 Aceptado: 6 de noviembre del 2015

Cómo citar este artículo: HernándezBernal, P. (2015). Alternativas de modelos de negocios para las mipymes colombianas desde la responsabilidad social empresarial, el comercio justo, los negocios inclusivos y el valor compartido. Cooperativismo y Desarrollo, 23(107), xx-xx. doi: http://dx.doi. org/10.16925/co.v23i107.1251

\section{Resumen}

Propósito: el objetivo de este artículo es proponer una alternativa estratégica a los empresarios de las mipymes y a los nuevos emprendedores colombianos, a partir de la responsabilidad social empresarial (RSE), el comercio justo, los negocios inclusivos y el valor compartido, como opción de nuevas formas de producción y creación de desarrollo. Descripción: se presenta de forma independiente cada uno de los temas al realizar un recorrido sucinto, con el fin de sensibilizar al lector en relación con las nuevas prácticas de hacer negocios. Punto de vista: se considera pertinente y oportuno establecer una reflexión académica, la cual lleva a la visualización de los campos de acción de la RSE en el caso colombiano. Es así como es necesaria una investigación que, en primera instancia, brinde la claridad sobre las raíces teóricas de la RSE, y, al mismo tiempo, se construya un marco teórico, en el cual sea posible identificar los conceptos con los que guarda relación. Conclusiones: el compromiso de los nuevos empresarios o empresarios renovados debe ser pensar en el progreso de la calidad de vida de sus empleados y el buen vivir de sus familias, logística inversa y sostenibilidad de la cadena de suministro, entre otros aspectos.

Palabras clave: asociatividad empresarial, comercio justo, negocios inclusivos, mipymes, responsabilidad social empresarial (RSE), valor compartido. 


\title{
Alternate business models for Colombian msmes based on corporate social responsibility, fair trade, inclusive businesses and shared value
}

\begin{abstract}
Purpose: The objective of this article is to propose a strategic alternative to MSMEs and new Colombian entrepreneurs, based on corporate social responsibility (CSR), trade fair, inclusive business and shared value, as an option for new forms of production and development. Description: Each of the topics is discussed independently through a brief overview in order to make the reader aware of the new practices of doing business. Point of view: It is considered relevant and timely to establish an academic reflection that leads to visualize the action fields of CSR in the Colombian case. Thus, research is needed to, first, provide clarity about the theoretical roots of CSR and, at the same time, build the theoretical framework in which it is possible to identify the concepts it relates to. Conclusions: The commitment of new or renewed entrepreneurs should be thinking about the progress in their employees' quality of life and the good living of their families, reverse logistics and supply chain sustainability, among others.
\end{abstract}

Keywords: business associations, fair trade, inclusive businesses, MSME, corporate social responsibility (CSR), shared value.

\section{Alternativas de modelos de negócio para as MPMEs colombianas a partir da responsabilidade social empresarial, do comércio justo, dos negócios inclusivos e do valor compartilhado}

\section{Resumo}

Propósito: o objetivo deste artigo é propor uma alternativa estratégica aos empresários das MPMEs e aos novos empreendedores colombianos a partir da responsabilidade social empresarial (RSE), do comércio justo, dos negócios inclusivos e do valor compartilhado, como opção de novas formas de produção e criação de desenvolvimento. Descrição: apresenta-se de forma independente cada um dos temas ao realizar um percorrido sucinto, a fim de sensibilizar o leitor quanto às novas práticas de fazer negócios. Ponto de vista: considera-se pertinente e oportuno estabelecer uma reflexão acadêmica, que leva à visualização dos campos de ação da RSE no caso colombiano. Assim, é necessária uma pesquisa que, num primeiro momento, ofereça a clareza sobre as raízes teóricas da RSE e, ao mesmo tempo, construa um referencial teórico em que seja possível identificar os conceitos com os que se relaciona. Conclusões: o compromisso dos novos empresários ou empresários renovados deve ser pensar no progresso da qualidade de vida de seus empregados e no bem viver de suas famílias, logística inversa e sustentabilidade da cadeia de abastecimento, entre outros aspectos.

Palavras-chave: associatividade empresarial, comércio justo, negócios inclusivos, MPMEs, responsabilidade social empresarial, valor compartilhado. 


\section{Introducción}

En las últimas décadas, el concepto de responsabilidad social empresarial (RSE) ha cobrado un enorme auge a nivel global y, en el caso particular de Colombia, cobra una importancia fundamental. Ha sido estudiado principalmente por los grupos de investigación universitarios, desde los cuales el sector empresarial ha sido motivado para comprender e implementar este concepto por medio de estrategias administrativas que permitan obtener resultados, tanto al interior de las empresas, como en la comunidad en las que se localizan. Por tanto, el concepto de RSE es conocido en el contexto colombiano. Sin embargo, su dinámica actual demanda profundización teórica y análisis de su accionar en términos de aplicabilidad.

Por lo anterior, se considera pertinente y oportuno establecer una reflexión académica, la cual lleve a la visualización de los campos de acción de la RSE en el caso colombiano. Es así como es necesaria una investigación que, en primera instancia, brinde la claridad sobre las raíces teóricas de la RSE y, al mismo tiempo, se construya un marco teórico en el que se puedan identificar los conceptos con los que guarda relación y fortalecen la implementación de la responsabilidad de las empresas en la comunidad a la que atienden, tales como son el comercio justo, los negocios inclusivos y el valor compartido.

De esta manera, se propone que las micro, pequeñas y medianas empresas (mipymes) conciban y visualicen la RSE como una herramienta estratégica para la contribución del sector privado en temas como la mitigación de necesidades socioeconómicas y la potencialización de las relaciones con los grupos de interés, en términos de mejoramiento continuo, todo lo cual resulte en perdurabilidad.

\section{Responsabilidad social empresarial}

\section{Recorrido epistemológico del concepto de RSE}

Primero es necesario entender el concepto de RSE y saber que no es una consideración nueva en el mundo empresarial. Desde la época de la Revolución industrial, se debe reconocer que se dio la industrialización desde la necesidad de mejorar la calidad de vida de los obreros y los empresarios terratenientes de la época. Esto trajo consigo consecuencias sociales marcadas de desigualdad y desequilibrio social, pero realmente el objetivo inicial era el mejoramiento de la calidad de vida de la humanidad, buscando hacer de sus procesos manuales procesos industriales y convertir la mano de obra en algo menos artesanal.

En nombre del desarrollo humano, también las teorías clásicas y científicas de la administración de Taylor y Fayol buscaron hacer de las industrias espacios de igualdad basados en la eficiencia ${ }^{1}$ y la disciplina como principios de perdurabilidad empresarial. Sin embargo, la teoría no se llevó a cabo en la totalidad de las empresas y, desde entonces, se denomina la época de la producción no humana ${ }^{2}$, la cual tuvo como consecuencia también la teoría de relaciones humanas de Elton Mayo. Esta última llevó a los sindicatos a buscar y repensar los procesos de contratación laboral, los horarios de trabajo, la estabilidad laboral y el salario mínimo legal, entre otros. Por lo tanto, en términos generales, se observa como realmente en relación al mejoramiento empresarial y de la humanidad se han propuesto teorías que en su momento social y económico funcionan, pese a las consecuencias propias de ello.

La aplicación de RSE requiere que los empresarios repiensen sus modelos organizacionales. El primer parámetro de la RSE se dio con la ley antimonopolio de Sherman en la década de los ochenta del siglo XIx, la cual evidenció la necesidad de regular y controlar el entorno empresarial, protegiendo los intereses de la sociedad. Necesariamente surge en la década de los treinta del siglo xx y después de la Segunda Guerra Mundial, en 1945, cuando se emiten regulaciones por parte de entes supranacionales con el fin de proteger el medioambiente. Ya en 1980, la RSE aparece de manera voluntaria, envuelta en un contexto social de grandes contrastes entre la sociedad y las empresas, evidenciado en un alto consumismo y la desregulación del comercio. Esto hacía que los efectos negativos no fueran solo locales, sino globales, de manera que propende hacia el desarrollo, el crecimiento y mejoramiento de la calidad de vida de un

\footnotetext{
1 Entendida como la disminución de los errores que se cometen en la empresa sin tener en cuenta el tiempo y los recursos, temas que se desarrollan en la contemporaneidad con los nombre de eficacia y efectividad.

2 Concepto desarrollado por Peter Drucker (1996), en el ensayo de desafíos para la empresa del siglo xxi.
} 
grupo social específico, a fin de generar consecuencias positivas de sus actos.

\section{Trayectoria del concepto de RSE, desde la contemporaneidad}

De acuerdo con Bowen (1953), la RSE "se refiere a las obligaciones de los empresarios para aplicar políticas, tomar decisiones o seguir las líneas de acción que son deseables en términos de los objetivos y valores de nuestra sociedad" (p. 6). Es decir, el objetivo de las empresas debe ser servir a la sociedad y maximizar los beneficios, lo cual corrobora entonces que la función principal de la empresa es de naturaleza económica, mientras que se desarrolla por medio de procesos socialmente responsables.

Con referencia a lo anterior, Heald (1957) afirma que debe existir una "conciencia corporativa", la cual se refiere a que es obligación de la empresa servir a la sociedad, propendiendo hacia el desarrollo humano y la construcción de políticas. No obstante, posteriormente Frederick (1960, p. 60) concluye que hay tres ideas de la RSE destacadas: la idea del administrador público; el equilibrio de la competencia por los recursos corporativos; y la filantropía empresarial como el apoyo de las empresas a las buenas causas.

En palabras de McGuire (1963):

La idea de responsabilidad social supone que la corporación no solamente tiene obligaciones económicas y jurídicas, sino también ciertas responsabilidades a la sociedad que van más allá de estas. Esto quiere decir que las empresas deben actuar con justicia como un ciudadano correcto debería hacerlo (p. 144).

Es decir, la corporación empieza a tomar conciencia del impacto que tiene su desarrollo económico.

Aproximadamente, en 1970, surge un argumento en contra de la RSE por parte de Friedman (1970), que afirma que "la única responsabilidad de la empresa hacia la sociedad es la maximización de beneficios a los accionistas dentro del marco legal y ético del país". En 1979, como contraargumento, Carroll (1991) identifica que el concepto de RSE es integral, pues "abarca las expectativas económicas, legales, éticas, y discrecionales que la sociedad tiene de las organizaciones en un punto dado del tiempo" (p. 50).

Después de los decenios de 1960 y 1970, se dan avances frente a los conceptos de política empresarial, ética empresarial y teoría de stakeholders (Carroll, 1991). Sin embargo, se dan avances en el concepto de RSE hasta 1980, cuando afirma Drucker (1984): "Responsabilidad social es convertir un problema social en oportunidad económica y beneficio económico, en capacidad productiva, en habilidades humanas, en trabajos bien pagos y en riqueza" (p. 10). A continuación, en 1990, Carroll (1991, p. 43) plantea su definición de RSE, incluyendo en esta que la empresa debe cumplir con todas sus responsabilidades (económicas, legales, éticas y filantrópicas).

En los últimos años entran nuevas categorías en el concepto de RSE, tales como desarrollo sostenible, ética en los negocios y negocios inclusivos, entre otros. Es por esto que la Comisión de las Comunidades Europeas (2001) define la responsabilidad social como:

Un concepto con arreglo al cual las empresas deciden voluntariamente contribuir al logro de una sociedad mejor y un medio ambiente más limpio. Ser socialmente responsable no significa solamente cumplir plenamente las obligaciones jurídicas, sino también ir más allá de su cumplimiento, invirtiendo "más" en el capital humano, el entorno y las relaciones con los interlocutores. La experiencia adquirida con la inversión en tecnologías y prácticas comerciales respetuosas del medio ambiente sugiere que ir más allá del cumplimiento de la legislación puede aumentar la competitividad de las empresas (p. 4).

Para el 2006, Porter y Kramer (2006) afirman que "percibir la responsabilidad social como la construcción de valor compartido, y no como un control de daños o una campaña de relaciones públicas, requerirá un pensamiento de negocios radicalmente distinto" ( $\mathrm{p}$. 15). A partir de la definición propuesta anteriormente y de acuerdo con el concepto de RSE de la Comisión Europea (2001), presentada en el Libro Verde ${ }^{3}$, se determinan como las definiciones que enmarcan completamente la intención y alcance de la RSE.

\section{Universalización de la RSE}

Desde hace ya varios años han aparecido en el mundo empresarial un considerable número de normas técnicas, certificaciones, informes y memorias, entre

\footnotetext{
3 Los Libros Verdes son documentos publicados por la Comisión Europea, cuyo objetivo es estimular una reflexión a nivel europeo sobre un tema concreto. En ellos se invita a las partes interesadas (organismos y particulares) a participar en un proceso de consulta y debate sobre las propuestas que presentan.
} 
otros mecanismos cuyo propósito es implementar y divulgar programas de RSE. Esto en razón a que grupos de interés han presionado por conocer fuera de los ámbitos financieros el ejercicio de su RSE.

No obstante, de acuerdo con Strandberg (2010, p. 7), en esa tarea de informar las empresas empezaron a encontrarse con el problema de cómo medir las acciones de RSE y sus resultados. Por tal motivo, surgen y cobran gran importancia varias normas, guías y estándares en general, entre las que se destacan:

- $\quad$ El Pacto Mundial:

Es una iniciativa internacional que promueve implementar 10 Principios universalmente aceptados en las áreas de Derechos Humanos; es un marco práctico para desarrollar, implantar y divulgar políticas y prácticas de sostenibilidad corporativa, ofreciendo a sus firmantes herramientas de gestión para ayudarles a implementar modelos de negocio sostenible (Pacto Mundial, 2013).

- Global Reporting Initiative (GRI):

Es una organización creada en 1997 por la convocatoria de la Coalición de Economías Responsables del Medio Ambiente (CEREs) y el Programa de Medio Ambiente de las Naciones Unidas (PNUMA). El GRI ha desarrollado la 'Guía para la elaboración de un informe de sostenibilidad', su misión es mejorar la calidad, rigor y utilidad de los reportes de sustentabilidad para que alcancen un nivel equivalente al de los reportes financieros (Global Reporting Initiative Organization, 2012).

- SA 8000: “Es una certificación voluntaria la cual fue creada por una organización estadounidense llamada Responsabilidad Social Internacional (Social Accountability International-SAI), con el propósito de promover mejores condiciones laborales" (Organización de las Naciones Unidas para la Alimentación y la Agricultura [FAO], 2007).

- AA 1000:

Son normas basadas en principios para ayudar a las organizaciones a ser más responsables y sostenibles. Las normas AA1000 están diseñadas para el pensamiento integrado requerido por el bajo contenido de carbono y la economía verde, y el apoyo de informes y validación integrados (AccountAbility Organization, 2012).
- ISO 26000:

Norma que pretende agregar valor al trabajo existente sobre la responsabilidad social (Rs), mediante la extensión de la comprensión y la implementación de la Rs mediante: El desarrollo de un consenso internacional sobre lo que significa Rs y los asuntos de Rs que las organizaciones necesitan abordar. El aporte de una guía para la traducción de los principios en acciones efectiva. La afinación de las mejores prácticas que ya han evolucionado y la difusión de la información en todo el mundo para el bien de la comunidad internacional (ISO, 2010).

Según lo planteado por Strandberg (2010, p. 13), en el 2005, el $52 \%$ de las principales 250 compañías de la lista Fortune 500 publicaron informes de RSE. La mayoría de esas empresas pertenecen a los sectores con impacto ambiental significativo, tales como las industrias extractivas, químicas o manufactureras. Asimismo, el número de bancos y compañías de seguros que publican esos informes se está incrementando. Puede observarse entonces que la elaboración y divulgación de informes y memorias de RSE han dejado de ser una moda, para convertirse en un requisito a fin de alcanzar una gestión comercial y social adecuada en las empresas.

La gestión de la RsE puede y debe ser evaluada. La medición es una tarea fundamental que permite conocer el grado de cumplimiento de los objetivos empresariales, con el fin de implementar estrategias encaminadas a cumplir las metas que tengan las empresas en ese sentido. Dado que cada empresa es diferente, es difícil establecer indicadores que evalúen en realidad la gestión de la RSE, y sean además estandarizados. Por lo tanto, algunas compañías los usan como estrategia de marketing, o en ocasiones ajustan estos informes de gestión, con el fin de parecer más responsables de lo que en realidad son.

Strandberg (2010, p. 13) establece que los indicadores se establecen bajo dos formas. La primera son aquellos que son desarrollados por la propia empresa, y los segundos son indicadores establecidos bajo normas de medición y comparación que ya se encuentran previamente establecidas en los estándares descritos anteriormente. Dichas normas proporcionan una serie de requisitos básicos, los cuales son utilizados como base para estructurar la comunicación de los indicadores eficazmente, con el propósito de ofrecer credibilidad a la empresa. Por otro lado, si la empresa 
es quien establece dichas normas, se encuentra en la obligación de explicar cómo se identifica el indicador y de qué manera es medido.

Los indicadores de RSE tienen como objetivo medir los impactos de las diversas actividades empresariales, tanto en la sociedad, como en el medio ambiente, y así instaurar acciones a tomar en aquellos resultados negativos. Estos indicadores pueden ser cualitativos o cuantitativos o, según Strandberg (2010), indicadores de triple resultado (social, económico y ambiental). En ocasiones, la interpretación de los resultados de los indicadores produce subjetividad, por lo cual se crea dificultad a la hora de establecer claridad y pertinencia en los diferentes proyectos y resultados de las diversas acciones de RSE que la empresa desea comunicar.

Según Zadek (2005) y Ligteringen (2005), el uso de los indicadores de RSE tiene dos motivos. Primero, ayudan a gestionar e implementar prácticas más responsables y trasparentes; segundo, proveen una visión clara acerca de los conceptos de desarrollo sostenible y RSE. A través de ellos, se pretende establecer que la empresa y sus empleados realicen actividades acordes con los indicadores, encontrando un comportamiento sostenible del personal y una integración de los objetivos en función de la organización.

Strandberg (2010, p. 13) también argumenta como las normas técnicas son una regla que se debe seguir, y a la cual las conductas, tareas y actividades de la empresa se deben ajustar, para así facilitar el inicio, la implementación y el cumplimiento de los proyectos de RSE en las compañías. Sin embargo, también es importante resaltar que al existir un gran número de normas, las empresas no saben distinguir las unas de las otras, por lo cual es una gran desventaja pues se estaría dejando de lado la pertinencia del uso de cada una, o bien puede suceder que no se estarían implementando de manera correcta. Por lo tanto, planear, implementar, medir y publicar los resultados de los indicadores de RSE es de vital importancia, así como establecer canales honestos y verídicos de comunicación con los grupos de interés (stakeholders), pues son estos los que establecen el éxito o fracaso de las actividades relacionadas con la RSE:

En la medida en que las empresas se comprometan con la RSE y trabajen en conjunto con todos los stakeholders, cualquier iniciativa que adopten será exitosa; de lo contrario, no será más que otra empresa tratando de unirse a la moda de publicar informes de RSE (Duque, Cardona y Rendón, 2013).

A continuación, en la tabla 1 , se enumeran algunos de los muchos avances que, a nivel mundial, se han hecho sobre la RSE, y se citan en este documento con el fin de que los interesados indaguen sobre ellas y observen como existen manuales y guías que coadyuvan a hacer el proceso posible.

Tabla 1

Certificaciones $y$ avances en RSE

\begin{tabular}{ll}
\hline AÑO & \multicolumn{1}{c}{ AVANCE TÉCNICO } \\
\hline 1987 & Informe Brundtland \\
\hline 2000 & Lanzamiento del Global Compact \\
\hline 2002 & Lanzamiento del Libro Verde \\
\hline 2002 & Reportes de sostenibilidad obligatorios en Francia \\
\hline 2004 & El GRI se convierte en una institución permanente \\
\hline 2004 & $\begin{array}{l}\text { Lanzamiento de African Sustaintanility Business } \\
\text { mericana de RSE }\end{array}$ \\
\hline 2005 & $\begin{array}{l}\text { Grupo de trabajo para crear standar internacional, } \\
\text { Próxima Iso 26000 }\end{array}$ \\
\hline 2008 & $\begin{array}{l}\text { sGE de la ocDE, sistema de gestión ética y socialmente } \\
\text { responsable. }\end{array}$ \\
\hline 2010 & Iso 26000 última versión \\
\hline 2011 & $\begin{array}{l}\text { Normas de aseguramiento para entrega de informes } \\
\text { RS-AAA 1000. }\end{array}$ \\
\hline 2012 & sAI 8000, social accountability internacional y la oit. \\
\hline 2013 & Guía del pacto mundial, Naciones Unidas. \\
\hline
\end{tabular}

Nota. Elaboración propia a partir La RSE en el contexto internacional y la norma Iso 26000 de Responsabilidad Social, por D. Pesce, 2006.

La RSE por sí sola ayuda al empresario a autoevaluarse con el fin de generar mejores procesos gerenciales, teniendo en cuenta que no es una obligación legal, sino una opción que se presenta hoy en día ante las necesidades del entorno y, por lo tanto, es ante esta estrategia que las empresas deciden generar mayor valor para sus grupos de interés, además de lo que hacen en su propósito económico y comercial. Es por esto que ahora cabe darle paso a un concepto que bien puede ser el ejemplo perfecto de cómo debe ser la práctica responsable, razón por la cual se presenta a continuación el origen del comercio justo. 


\section{Comercio justo}

\section{Desarrollo epistemológico del concepto de comercio justo}

El origen del comercio justo se sitúa hacia 1964, cuando durante la conferencia de las Naciones Unidas sobre el Comercio y Desarrollo (UNCTAD) los países del sur allí presentes alegaron que el "comercio, no ayuda". Estos países del sur pedían relaciones comerciales más justas en el ámbito internacional, que les permitieran un desarrollo económico autónomo, en lugar de ser objeto de políticas de cooperación o ayuda internacional. Según Sichar (2002), el comercio justo es una alternativa al comercio tradicional y busca promover el consumo por medio de distintas vías y productos, de acuerdo con criterios económicos, así como con valores sociales y ecológicos.

De esta manera, Sichar (2002) también señala que el comercio justo recupera el vínculo entre productor y consumidor, de tal forma que "desaparecen los intermediarios; el consumidor obtiene, junto con el producto, información del productor, lo que permite conocer lo que se está comprando". Otra de las vías de apoyo es para que "los campesinos y pequeños productores de zonas empobrecidas encuentren el cauce para vivir dignamente de su trabajo" (De la Torre, 2004, p. 10), de tal manera que reciban un precio adecuado por sus productos, el cual se considere con respecto al nivel de calidad de vida de su país. Por otra parte, los consumidores obtendrán productos de calidad, con garantía de que se han respetado los derechos de los trabajadores y el medio ambiente. Se reconocen así, como un valor intrínseco al producto. Igualmente, Sichar (2002) informa que una de las condiciones que debe cumplir el comercio justo es que "las organizaciones productoras deben destinar parte de sus beneficios a las necesidades básicas de sus comunidades: salud, educación y vivienda, entre otros".

En la actualidad, el comercio internacional es cada vez más injusto, ya que los intercambios comerciales entre países industrializados y países en vía de desarrollo aumentan en volumen, lo cual desafortunadamente no propende a la equidad entre naciones y hace esquiva la idea de justicia e igualdad. Por ejemplo, los países industrializados no han permitido a los países en vía de desarrollo una comercialización de productos manufacturados, por lo que estos solo se especializan en productos del sector primario. De igual manera sucede en Colombia, en donde la diferencia entre las ciudades y municipios es notoria, tanto en términos industriales de innovación, ciencia y tecnología, como en los precios de los productos.

El economista Ha Joon Chang (2004) describe, en Retirar la escalera, la manera en que los países del primer mundo imponen barreras al libre comercio. El autor se refiere al libre comercio como "la libre circulación del capital y el trabajo" y argumenta "que es la clave de la prosperidad general” (p. 1). A partir de esta premisa, el autor explica cómo los países han tomado medidas de intervencionismo, a fin de impedir la entrada de productos extranjeros a su territorio, mientras exigen de otros países la eliminación de todo tipo de barreras para la entrada de sus productos.

Estos países apoyaban su industria por medio de subsidios, mediante los cuales hacían que sus productos fueran más baratos en comparación con los de los demás países, y así poder inundar los mercados internacionales con sus productos, obligando al resto de los países a dedicar su producción a productos agrícolas y de materias primas. Estas potencias, además de interponer barreras a la entrada de productos y regular parte del mercado internacional, impiden que en los países en vía de desarrollo se otorguen subsidios a la industria o al campesino, y así logran que sus productos sean más competitivos al inundar los mercados de la periferia.

Respecto a esto, Ha Joon Chang, en una conferencia sobre América Latina, sugirió:

Si los subsidios son buenos o malos, depende. En ciertos casos, por ejemplo, los subsidios ayudan a erradicar el trabajo infantil. Además, la pobreza es un serio problema en América Latina, por eso, no veo nada de malo en que se destinen subsidios a servicios básicos de educación y salud. Pero tener demasiados subsidios vuelve difícil el manejo del presupuesto, sobre todo si el Estado no genera ingresos para cubrir esos egresos adicionales (Zambrano, 2011).

Esto hace evidente la necesidad de identificar el comercio justo como una faceta ambigua de la economía contemporánea, y un cuestionamiento a la naturaleza moral del capitalismo (Smith, 1759).

De otra parte, Barrat Brown, en Comercio justo, comercio injusto (1998), afirma que, a pesar de la reducción general de las medidas protectoras impuestas por los países desarrollados, estos - en particular, Estados Unidos y la Unión Europea-aplican arbitrariamente una protección que incluye cláusulas 
de salvaguardia, medidas antidumping o normativas sobre el origen de las mercancías. Con respecto a las barreras de restricción impuestas por los países del norte, las cuales llevan al incremento de los precios debido a estas políticas, el autor argumenta que:

No es fácil convencernos para que demos algo más de lo que en realidad debemos pagar. La caridad puede ser un motivo o, al menos un simulacro del deseo de comportarnos con los demás como quisiéramos que lo hicieran con nosotros (Barrat Brown, 1998, p. 35.).

De esta forma, se puede ver cómo la opinión de Barrat y Joon Chang coinciden en cuanto a las políticas de opresión que son impuestas por los países industrializados, dando lugar a lo que ellos denominan, injusticia comercial.

Asimismo, Barrat Brown (1998) informa que los consumidores podrían llegar a pagar más por un producto, siempre y cuando el producto sea excelente y que dicha cantidad pagada irá a parar en manos de los productores originales. Dichos ingresos extra deben reinvertirse en mejorar las condiciones de todos los productores.

En una línea de análisis similar, James Bovard considera que el comercio justo es uno de los grandes fraudes del siglo xx. Esto lo deduce al revisar las políticas de proteccionismo que impone Estados Unidos al comercio, y las altas tasas a las importaciones. Para Bovard (1991), el comercio justo de Estados Unidos, por lo general, significa: "intervención del gobierno para dirigir, controlar o restringir el comercio". Además, argumenta que el proteccionismo produce la corrupción política, el estancamiento económico y el conflicto internacional. Sin embargo, muchas personas insisten en que, a pesar de que el proteccionismo dificulta la capacidad de una nación para alimentar, vestir y generar proyectos de vivienda, las ganancias morales del proteccionismo son mayores que las pérdidas económicas.

Al observar las restricciones y el favorecimiento de los países del norte a sus industrias, el autor David Ransom (2011) expresa que, en el comercio, todo es válido, con tal de ganar, y que la moralidad en este campo de la actividad humana no existe. Además, explica que los únicos países que se ven beneficiados por el comercio son los ricos y poderosos, lo que ratifica su naturaleza injusta. El autor, sin embargo, considera que el comercio justo en un sentido más amplio es una alternativa que puede florecer con mayor fuerza.
Finalmente, Vivas y Montagut (2006) proponen el fortalecimiento de un movimiento crítico al estado del intercambio económico en la actualidad, y no hacer del comercio justo un simple instrumento de marketing al servicio de las empresas. En su trabajo, los autores buscan demostrar que el comercio justo no es una herramienta para aliviar conciencias y cómo crear moralidad en las personas a través del pago de un precio más alto a favor de los campesinos del sur no es suficiente. El comercio justo, en su lugar, es una apuesta por mejorar las injusticias que afectan al norte y al sur; los autores señalan que en el comercio justo existen dos polos de referencia:

El sector 'tradicional y dominante' considera al comercio justo como una transacción 'Norte-Sur', mientras que el polo 'global y alternativo' señala que el comercio justo debe ser también 'Norte-Norte' y 'Sur-Sur'. Esto significa que tanto el Norte como el Sur deben avanzar hacia las relaciones comerciales más justas y solidarias, que sólo serán posibles con un modelo de agricultura, distribución y consumo ligado al territorio (p. 24).

Luego de considerar los argumentos y conceptos de los distintos autores acerca del comercio justo y sus implicaciones, esta investigación propone el abordaje descriptivo de la forma como el comercio justo juega en dos escenarios con una lógica de moral distinta.

Finalmente, este sucinto recorrido teórico entre la RSE y el comercio justo, se propone darle la suficiente importancia al desarrollo local, como lo plantea Krugman (2006, pp. 17-21), en el que los países en vía de desarrollo deben preocuparse, y ocuparse en desarrollar su propio comercio; es decir, en desarrollar proyectos económicos locales, más que globales.

Por lo anterior, a continuación se proponen alternativas de desarrollo económico local, con el fin de fortalecer las condiciones actuales de los negocios.

\section{Tendencias del comercio justo para los consumidores}

Indagando fuentes secundarias para la realización de este artículo, se pretende mostrar a los lectores cifras sensibles frente al concepto de comercio justo, para luego citar estudios de fuentes primarias que describan el impacto que este tiene en el mundo empresarial.

Primero, en el estudio realizado por la Organización Price Water House Coopers (PWHC), 
para el 2012, a más de 1000 compradores en América Latina, en la cual se puede evidenciar claramente cómo un consumidor toma la decisión de dejar de comprar un producto o adquirir un servicio por diversas causas, tal como se observa en la tabla 2 .

Tabla 2

Principales causas por las que el consumidor dejaría de comprar

\begin{tabular}{lll}
\hline \multicolumn{3}{c}{ Dejaría de Comprar por: } \\
\hline \multicolumn{1}{c}{ Frecuencias } & $\%$ \\
\hline $\begin{array}{l}\text { Mano de obra } \\
\text { infantil }\end{array}$ & 140 & $13 \%$ \\
\hline Abusos laborales & 129 & $12 \%$ \\
\hline $\begin{array}{l}\text { Pruebas con ani- } \\
\text { males }\end{array}$ & 89 & $8 \%$ \\
\hline $\begin{array}{l}\text { Falta de respeto } \\
\text { de los derechos } \\
\text { humanos }\end{array}$ & 146 & $14 \%$ \\
\hline $\begin{array}{l}\text { Discriminación de } \\
\text { género }\end{array}$ & 101 & $9 \%$ \\
\hline $\begin{array}{l}\text { Publicidad enga- } \\
\text { nosa }\end{array}$ & 135 & $13 \%$ \\
\hline $\begin{array}{l}\text { Falta de esfuerzo } \\
\text { por contratar } \\
\text { discapacitados }\end{array}$ & 86 & $8 \%$ \\
\hline $\begin{array}{l}\text { Falta de infor- } \\
\text { mación para la } \\
\text { inversión }\end{array}$ & 75 & $7 \%$ \\
\hline $\begin{array}{l}\text { Corrupción, frau- } \\
\text { des y malos mane- } \\
\text { jos financieros }\end{array}$ & 96 & \\
\hline $\begin{array}{l}\text { Abuso de poder } \\
\text { por parte de los } \\
\text { directivos }\end{array}$ & 43 & \\
\hline $\begin{array}{l}\text { No involucrar a los } \\
\text { proveedores en la } \\
\text { cadena de valor }\end{array}$ & 27 & \\
\hline & & \\
\hline
\end{tabular}

Nota. Elaborada a partir de Price Water House Coopers. Disponible en http://www.pwc.com/co/es/publicaciones.html.

Es relevante entonces la claridad que se ha ido adquiriendo frente al concepto de compra. Las empresas trabajan ya no solamente por ser reconocidas, sino más queridas entre sus compradores.

Entre tanto, la organización COMUNICA-RSE, en el 2013, indaga sobre cómo afecta la reputación de la empresa en el momento de la compra de un bien o servicio, según la edad del comprador, para una escala de 100 compradores.

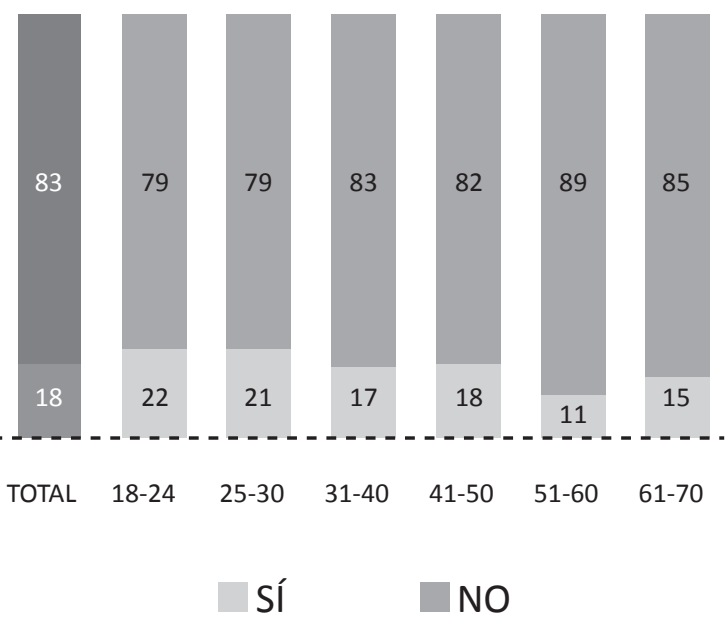

Figura 1. Mala reputación de la empresa, decisión de no comprar por rango de edad.

Se puede deducir que, independientemente de la edad del comprador, en promedio el $80 \%$ de los compradores dejarían de adquirir bienes o servicios si la empresa tiene mala reputación o no cumple con los compromisos propios de la RSE y el comercio justo.

De la comparación mundial que se realiza en el Estudio Mori-2000 Environics (Canadá) para 20 países, se pueden rescatar para este artículo tres preguntas, así:

Tabla 3

Estudio Mori - 2000 Environics (Canadá)

\begin{tabular}{|c|c|c|}
\hline $\begin{array}{c}\text { ¿Cuáles son los aspectos que la pobla- } \\
\text { ción toma en cuenta a la hora de juzgar } \\
\text { una compañía? }\end{array}$ & Chile\% & EEUU\% \\
\hline Marca y reputación & 41 & 26 \\
\hline Las prácticas laborales y ética empresarial & 12 & 28 \\
\hline $\begin{array}{l}\text { La responsabilidad demostrada a la } \\
\text { sociedad }\end{array}$ & 26 & 21 \\
\hline El impacto medioambiental & 9 & 16 \\
\hline \multirow{3}{*}{$\begin{array}{l}\text { ¿Respeta a las empresas conocidas por } \\
\text { compensar generosamente a las comuni- } \\
\text { dades en que operan? }\end{array}$} & Suecia & $79 \%$ \\
\hline & Chile & $78 \%$ \\
\hline & España & $69 \%$ \\
\hline \multirow{3}{*}{$\begin{array}{l}\text { ¿Ha castigado a una emprepsa por no } \\
\text { ser socialmente responsable? }\end{array}$} & Chile & $5 \%$ \\
\hline & Canadá & $14 \%$ \\
\hline & España & $9 \%$ \\
\hline
\end{tabular}

Nota. Elaboración propia. 
Esto indica cómo cada vez incursionar en mercados desarrollados o países de la Organización para la Cooperación y el Desarrollo Económicos (OCDE) será más riguroso, en cuanto RSE y comercio justo se trate. Ya no es solamente un tema de precio o calidad.

Y para Colombia, en la Asociación Nacional de Empresarios de Colombia (ANDI, 2012), también surge la necesidad de hacer una encuesta a los consumidores, por medio de la cual se puedan vislumbrar las tendencias de las exigencias de consumo y de servicios. Así se ve en el resumen que se realiza en la tabla 4.

Tabla 4

Estudio ANDI - 2013 (Colombia)

\begin{tabular}{ll}
\hline \multicolumn{1}{c}{ Comentarios } & \multicolumn{1}{c}{$\%$} \\
\hline No consumir productos socialmente negativos & 24 \\
Consumir los productos buenos solamente & 17,3 \\
Presentar quejas y reclamos a las empresas & 26,8 \\
Denunciar a las autoridades competentes & 22 \\
Denunciar a los edios de comunicación & 9,9 \\
\hline
\end{tabular}

Nota. Elaboración propia.

Es evidente que también los consumidores colombianos tienen una postura clara frente a los beneficios que se tienen al adquirir un bien o servicio. Las autoridades competentes y las denuncias frente al consumo empiezan a tener fuerza en el mercado.

\section{Negocios inclusivos}

\section{Revisión del origen del concepto de los negocios inclusivos}

La pobreza ha sido enmarcada como uno de los grandes problemas mundiales. Por esta razón, organizaciones como la onU consideran fundamental contar con programas de lucha que sean pertinentes para cada país, con un proceso paralelo por el que se cree un medio internacional favorable a este grupo de personas, los cuales pueden no ser un atractivo para las empresas, pero que sumados constituyen un potencial de negocios como socios, consumidores, proveedores y distribuidores.

El Consejo Empresarial Mundial para el Desarrollo Sostenible (WBCSD) es una organización dirigida por los principales gerentes que reúnen aquellas compañías con visión de impulsar a la comunidad empresarial a crear un futuro sostenible para la sociedad y el medio ambiente, y en el cual compartan sus conocimientos, experiencias y mejores prácticas trabajando con los gobiernos y organizaciones intergubernamentales con un único fin: hacer negocios inclusivos definidos como "soluciones sostenibles de negocio que expanden el acceso a bienes, servicios y oportunidades de ingresos para segmentos de bajos ingresos de manera comercialmente viable" (Red Empresarial de Negocios Inclusivos, s. f.).

Entre tanto, pero con el mismo compromiso y en alianza con el WBCSD, se encuentra la Netherlands Development Organization, cuya función es abarcar el crecimiento económico y el desarrollo de cadenas de valor, teniendo en cuenta los servicios financieros $y$, a su vez, educando de forma profesional y técni$\mathrm{ca}$, haciendo seguimiento a proyectos y programas en el sector de la agricultura, agua, energía renovable y otros. Es así como esta organización establece estrategias para entidades públicas, empresas privadas y organizaciones civiles, con el fin de dar soluciones que involucren a la población de bajos recursos e incentiven el aumento de sus ingresos en el propósito de lograr mejorar su calidad de vida.

En 1993, se diola creación del Consejo Empresarial Colombiano para el Desarrollo Sostenible (Cecodes), cuyas iniciativas empresariales se orientaron al desarrollo sostenible del país, y reunían elementos tales como el crecimiento económico, el progreso social y el balance ecológico.

De acuerdo con lo que informa Cecodes, se busca orientar a las empresas para que sus iniciativas sean económicamente rentables, ambiental y socialmente responsables. A fin de dar cumplimiento a esto, en el 2010, el Gobierno colombiano aprobó la Ley 70, cuyo objetivo plantea reconocer y valorar las actividades de responsabilidad social apadrinadas por las empresas dentro del territorio nacional con incentivos que retribuyeran dichas prácticas, de manera que se logre en el mediano plazo la vinculación de más empresas que fomenten la inclusión social, como objetivo dentro de su estructura empresarial.

El concepto de Negocios Inclusivos (NI) está vinculado a la generación de valor para la llamada base de la pirámide, y las transacciones con los "mercados de mayoría" que se constituyen. También ha sido definida de diferentes maneras, como lo hace la ONU, desde el uso de niveles de ingresos (que pueden o no coincidir con los niveles usados para definir pobreza y extrema pobreza), hasta el segmento socioeconómico de bajos ingresos que vive primariamente 
en el sector informal. El potencial de los NI de tener un impacto en la pobreza claramente está relacionado con la selección de la población meta del negocio, y si se reconoce o no como pobre. Sin embargo, al paso del tiempo y el desarrollo de estudios se incluye en procesos como los que implican proveedores y distribuidores, lo cual propicia una oportunidad para las empresas de reducir costos operativos y de producción, aumentando su nivel de cobertura o expansión.

Cecodes (2008) define los negocios inclusivos como una iniciativa empresarial que pretende generar ganancias incluyendo ciudadanos de bajos ingresos en su cadena de valor. Un negocio inclusivo se establece a través de una empresa llamada "ancla", y uno o más emprendedores o grupo de consumidores locales buscando maximizar el valor social y económico.

Entonces, para países como Colombia, donde los niveles de inequidad con el paso de los años son más altos y, según varios estudios de la Comisión Económica para América Latina (CEPAL), se encuentra dentro de los más altos de la región, cabe citar a Porter (2011), que aduce que la creación de valor se debe hacer a través del sector privado, el cual debe ampliar la mentalidad de la función que cumple en la prosperidad del país, siendo este el primer paso de valor compartido en el cual las empresas deben comprender que en los objetivos empresariales, se recomienda que las mipymes incluyan en su desarrollo económico el beneficio para la sociedad, haciendo así que estas sean económicamente sostenibles y perdurables con el paso del tiempo.

Como bien lo indican Porter y Kramer (2011), las empresas deben asumir el liderazgo para volver a unir los negocios con la sociedad. Es por esto que empresas privadas colombianas han decidido adoptar prácticas de responsabilidad social empresarial, las cuales inducen al desarrollo sostenible, argumentando que favorecen el bienestar social, la educación y la salud, generando empleo e impulsando el desarrollo local.

Sin embargo, desafortunadamente en Colombia la situación es diferente, ya que algunas multinacionales realizan negocios inclusivos con el fin de satisfacer intereses propios, sin importar la influencia que estos puedan tener en la población que involucran. Según escribe Freytas (2010) en "Tres críticas que se hacen a los Negocios Inclusivos", las empresas pagan salarios inequitativos a personas de bajos recursos, las cuales hacen parte de su cadena de valor reduciendo así sus costos de producción.
No obstante, en Colombia, existen algunas organizaciones que exponen el impacto que generan los negocios inclusivos, y cómo el país se encuentra en el ámbito de la responsabilidad social empresarial. En el 2008, Cecodes publicó un documento en el cual exponía las modalidades que las empresas habían puesto en práctica, a fin de lograr la adhesión en sus cadenas de valor de población con bajo poder adquisitivo, tomando como ejemplo empresas colombianas influyentes en la economía.

Además, en Colombia, se encuentra Bancoldex, ente que se ha convertido en un ejemplo de apoyo a las compañías, ya que ofrece servicios de financiación con tasas de interés bajas para quienes demuestran con información real y transparente las prácticas de inclusión de la población vulnerable, dentro de los procesos de su cadena de valor. Las mipymes que se acogen a lo dispuesto por dicha ley, reciben estímulos para impulsar su desarrollo, tales como puntajes adicionales en licitaciones públicas, facilidades de acceso a créditos, el acceso a programas de fomento económico y de innovación tecnológica, y demás beneficios que siguen trabajándose desde agremiaciones como la ANDI, Fenalco y el Ministerio de Comercio, ente otras.

Por otro lado, Freeman (1984) considera que las organizaciones deben estar compuestas por grupos de interés (en inglés, stakeholders), entre los cuales están incluidos inversionistas, colaboradores, suministradores, clientes, administración, la comunidad, el gobierno y la sociedad en general. Sin embargo, para Michael Porter, lo anterior no es suficiente, ya que "la responsabilidad clásica de los empresarios ni la filantropía responde con efectividad a las necesidades sociales" (Barcenas, 2015). Es por esto que, con el paso de los años, se ha creado un nuevo concepto de negocios inclusivos, en el cual se busca generar valor para las partes que en este intervienen a través de políticas y prácticas operacionales que logren mejorar la competitividad de una empresa, sin dejar a un lado la comunidad donde se opera, ayudándola a mejorar sus condiciones económicas y sociales.

Este concepto busca ir más allá de las utilidades generadas en el corto plazo a través de la implementación de la responsabilidad social empresarial, aplicando una estrategia de largo alcance que combine el lucro de la empresa con el bienestar social.

Es así como para países como Colombia, en donde los niveles de inequidad con el paso de los años son más altos y, según varios estudios de la CEPAL, se encuentra dentro de los más altos de la 
región. Sin embargo, Porter y Mark (2011) aducen que la creación de valor se debe hacer a través del sector privado, el cual debe ampliar la mentalidad de la función que cumple en la prosperidad del país, siendo este el primer paso de valor compartido en el cual las empresas deben comprender como en los objetivos empresariales se recomienda que las mipymes incluyan en su desarrollo económico el beneficio para la sociedad, haciendo así que estas sean económicamente sostenibles y perdurables con el paso del tiempo.

Por esto, los negocios inclusivos necesitan la participación conjunta de diferentes actores, de manera que cada uno de ellos aporte desde su competencia, conocimiento o especialidad, y desempeñe un papel importante en las fases de planeación, implementación y medición de los mismos.

Tabla 5

Beneficios de los negocios inclusivos

\begin{tabular}{ll}
\hline \multicolumn{2}{c}{ Como socios, proveedores o distribuidores } \\
Para la empresa & \multicolumn{1}{c}{ Para la población } \\
\hline - Seguridad de abasteci- & - Precios y condiciones justas \\
miento & - Ventas aseguradas \\
- Trazabilidad y control de & - Creación o espansión de \\
calidad de la materia prima & puestos de trabajo \\
- Menores costos de tran- & - Capacitación y asistencia \\
sacción & técnica \\
- Riesgos compartidos & - Transferencia de conoci- \\
- Acceso al conocimiento y a & mientos y de tecnología \\
redes locales & Acceso al financiamiento \\
- Mejores relaciones con el & - Participación en un \\
gobierno & ambiente empresarial y de \\
- Posicionamiento en nuevos & inversiones \\
mercados de comercio justo & \\
\hline
\end{tabular}

\section{Tipología de los negocios inclusivos}

Actualmente existen dos tipos de NI que proponen vincular población de bajos ingresos a las compañías: en primera medida, como socios, proveedores o creando empleo y transfiriendo habilidades y conocimiento, lo cual después se podrá consolidar como ventajas competitivas. La otra modalidad se refiere a los consumidores, esto es, productos de calidad que satisfagan las necesidades de la población a precios y condiciones que puedan ser accesibles para personas de bajos recursos.

Los NI nacieron con el objetivo de aliviar la pobreza creciente, al alinear los negocios de la empresa con las necesidades de empleo de la población, por lo cual se diseñó una relación de beneficio gana-gana en las dos modalidades, como se expone en la tabla 5 .

\begin{tabular}{lc}
\hline \multicolumn{2}{c}{ Como consumidores } \\
\hline \multicolumn{1}{c}{ Para la empresa } & \multicolumn{1}{c}{ Para la población } \\
\hline - Acceso a un nuevo mercado & - Mayor acceso a productos y \\
- Incremento de ingresos & servicios de calidad \\
- Transferencia de las innova- & - Precios menores y accesibles \\
ciones de los productos a los & - Mejor calidad de vida \\
mercados actuales & - Aumentos de productividad \\
- Aumento del valor y & \\
posicionamiento de la \\
marca para capturar nuevos \\
mercados
\end{tabular}

Nota. Tomado de Negocios inclusivos. Iniciativas empresariales rentables con impacto en el desarrollo, por SNV/WBCSD, 2008).

Como se puede observar en la tabla 5, los negocios inclusivos han sido creados como una estrategia en la cual las compañías siguen haciendo negocios y generando ganancias, pero con el propósito de crear bienestar para la población vulnerable y, al mismo tiempo, lograr desarrollo, crecimiento en la economía y en el país.

\section{Valor compartido}

\section{Origen del concepto de valor compartido}

El concepto de valor compartido - o también conocido como valor añadido- se vio impulsado principalmente en el artículo publicado en enero del 2011 por Michael Porter y Mark Kramer en Harvard Busines Review, "Creación de valor compartido: cómo reinventar el capitalismo y desatar una oleada de innovación y crecimiento". En este se expone principalmente como este término no tiene que ver ni con filantropía, ni responsabilidad social, ni sostenibilidad, sino que está relacionado con obtener un beneficio mayor en la empresas.

Porter y Kramer explican en su artículo cómo “el concepto de valor compartido reconoce que las necesidades sociales, y no sólo las necesidades económicas convencionales, son las que definen los mercados." (citados en Castaño, 2014). En general, denominan el concepto como la unión que debe tener tanto la 
empresa, como la sociedad, a fin de obtener un beneficio mayor, y común, claro está. Con la inclusión del valor compartido en las empresas, Porter y Kramer invitan principalmente a generar un beneficio a la sociedad.

De igual manera, en ese artículo se muestran tres formas de generar valor compartido. La primera es la reinversión o reconcebir los productos y mercados. Esta es importante ya que según los autores, para llegar a satisfacer necesidades en los mercados "subatendidos", se necesitan productos que, de una u otra forma, sean rediseñados o, de igual manera, se empleen diferentes métodos de distribución.

El segundo es la redefinición de la productividad en la cadena de valor. Consiste en entender cómo los problemas que de una u otra forma tiene la sociedad pueden llegar a establecer una serie de costos, los cuales son principalmente económicos en la cadena de valor que tiene la empresa en particular, y lo cual es necesario analizar para generar un beneficio tanto a la sociedad, como a la misma empresa.

Por último, está el desarrollo de clústeres de empresas locales. Cabe aclarar que los clústeres son un conjunto de empresas y/o instituciones relacionadas entre sí, las cuales pertenecen a un mismo sector o segmento de mercado con el fin de colaborarse entre sí para ser más competitivas. Tener esta interacción o ayuda permite que las capacidades locales sean más fuertes en diferentes áreas, las cuales pueden ir relacionadas con áreas generales como las de producción, ayudando a que estas tengan un mejor comportamiento. Y si no se cuenta con un clúster de apoyo adecuado, pueda que el área de producción sufra por el contrario un menor comportamiento y exista una caída en la misma.

Esta nueva definición de negocios y reinvención del capitalismo clásico propuesto por Porter y Kramer va más allá de hacer conscientes a las empresas de su contribución al bien general, así como del cambio de pensamiento que deben tener los ejecutivos y administradores de las empresas. Evidencia la importancia que tienen los clústeres en esta reinvención del capitalismo, ya que estas concentraciones de empresas no buscan acabar con la competencia como se proponía anteriormente- sino actuar bajo el principio según el cual trabajar juntos y mantenerse juntos es progreso, y así alcanzar un nuevo nivel de desarrollo e innovación.

En conformidad con lo anterior, Porter y Kramer también enfatizan que el óptimo de la economía y los temas sociales no tienen que ir en sentido opuesto, ya que cuando se crea valor dentro de la empresa, también debe compartirse ese valor con la sociedad. Por ello, no debe confundirse el valor compartido con responsabilidad social empresarial, y mucho menos con filantropía, pues el valor compartido lo que busca es cambiar a las empresas y sus formas de hacer negocios.

Según estos autores, para que pueda existir valor compartido, se deben conocer los problemas y las necesidades latentes de la comunidad. Al conocerse estas necesidades, es posible llegar a un nivel más alto de competitividad, ya que se pueden atacar los problemas de una manera más directa a través de las empresas $y$, por ende, se aumentan las utilidades, tanto para las instituciones, como para la sociedad. Por esta razón, Michael Porter manifiesta que, para alcanzar el valor compartido, debe existir esa sinergia entre las partes.

Asimismo, es importante destacar que Porter sostiene que las organizaciones de carácter social, e incluso el Estado, no deben tener en cuenta la relación costo-beneficio, pues este tipo de instituciones miden la culminación de sus programas a través de dinero y recursos, y debe ser a través de los valores obtenidos.

Vale la pena señalar cómo un claro ejemplo de creación de valor compartido se puede evidenciar en las llamadas empresas híbridas. Este tipo de empresas, además de generar utilidad, también aumentan el mejoramiento de las condiciones que tiene la sociedad.

Por otra parte, con el fin de incrementar el valor compartido, también se debe tener en cuenta la implementación, el desarrollo y el crecimiento de los grupos de industrias aglomeradas en el mismo espacio geográfico, llamadas también clústeres. Porter y Kramer (2011) enfatizan que el éxito de las instituciones se da por la infraestructura y demás compañías que están alrededor.

También es importante mencionar la productividad de la cadena de valor, ya que se debe incluir a todos los actores que se ven afectados o involucrados de alguna forma dentro de los procesos de la empresa. Una estrategia puede ser la implementación de proveedores de pequeñas empresas, acompañado de acciones en pro del medio ambiente.

Porter y Kramer (2011) sostienen que la relación entre las empresas y la sociedad tienen implicaciones muy profundas, pues desafortunadamente las empresas ya no se ven como soluciones para la sociedad. Por el contrario, se ven como problemas. De acuerdo con esto, hay que crear valor económico, 
el cual a su vez genere bienestar a la sociedad, más allá de los normales creados por una empresa. Por ello, se debe pasar de la filantropía a la responsabilidad social empresarial, y de ahí a la creación de valor compartido.

Para concluir, Porter y Kramer (2011) señalan que se debe usar el capitalismo para generar impacto social, ya que hoy en día este se usa como una mala palabra dentro de la sociedad. Las empresas deben empezar a cambiar sus mentalidades hacia el valor compartido, pues este ya es una realidad y es la nueva forma de alcanzar el éxito económico.

\section{Valor compartido vs. RSE}

Desde hace un tiempo, se ha evidenciado una visión negativa de la sociedad hacia el capitalismo, como causante de daños ambientales, sociales y económicos. La gran mayoría de empresas han centrado su interés en los rendimientos financieros a corto plazo, y algunas de estas han optado por hacer uso del concepto de RSE como una forma de justificar su accionar, más que por una convicción en los principios que este concepto plantea.

Por su parte, Friedman (1970), como representante del pensamiento neoclásico, consideró la obligación moral de la empresa únicamente bajo los beneficios de los accionistas, de manera que sostiene: "La maximización del beneficio es la responsabilidad social que tiene la empresa"; es decir, que la RSE incrementa los beneficios siempre y cuando la empresa actúe bajo sus libres mecanismos.

Indudablemente, siguiendo la idea de Porter y Kramer (2011), mientras que la CVC aprovecha los recursos únicos de una empresa para crear valor social y se convierte en parte integral de la rentabilidad y posicionamiento de una empresa, la RSE se enfoca principalmente en la reputación y tiene una conexión limitada con el negocio, partiendo de que no incluye la generación de estrategias económicas que potencialicen las compañías, haciendo que sea difícil la permanencia de la aplicación de RSE en el largo plazo.

A continuación, se proporciona una comparación entre estos dos conceptos en la tabla 6.

Tabla 6

Comparación entre responsabilidad social empresarial y valor compartido de acuerdo con Porter y Kramer

\begin{tabular}{ll}
\hline \multicolumn{1}{c}{ RSE } & \multicolumn{1}{c}{ CVC } \\
\hline Valor: hacer el bien & Valor: beneficios económicos y sociales en relación a los costos \\
$\begin{array}{l}\text { Ciudadanía, filantropía, sustentabilidad } \\
\text { Discrecional o en respuesta a la presión extrema }\end{array}$ & $\begin{array}{l}\text { Creación conjunta de valor entre la empresa y la comunidad } \\
\text { Ajena a la maximización de utilidadeds }\end{array}$ \\
$\begin{array}{l}\text { La agenda es determinada por los reportes hacia fuera y las prefe- } \\
\text { rencias personales }\end{array}$ & La agenda es específica de la empresa y se genera internamente \\
$\begin{array}{l}\text { Impacto limitado por la huella de la empresa y el presupuesto } \\
\text { RSC }\end{array}$ & Alinear de nuevo todo el presupuesto de la empresa \\
\hline
\end{tabular}

Nota. Elaboración propia a partir de "La creación de valor compartido", por M. Porter y M. Kramer, en Harvard Business Review, 2011. Recuperado de https://estrategiati.files.wordpress.com/ 2011/06/la-creacic3b3n-de-valor-compartido.pdf

\section{Asociatividad empresarial}

\section{Contextualización de asociatividad empresarial}

La asociatividad empresarial se conoce como un mecanismo de cooperación que surge entre las pequeñas y medianas empresas, en la cual cada uno de los agentes, ya sean empresas o personas, poseen una independencia jurídica, y de manera voluntaria deciden participar de modo conjunto con otros agentes. La creación de este fenómeno es imputado al enfrentamiento de los procesos de globalización presentado en las economías nacionales.

Los principales objetivos de este mecanismo de cooperación son: la adquisición de materias primas a un bajo costo en menos tiempo; desarrollo de tecnologías para el beneficio común; un mayor acceso a financiamiento que requiere garantías (las cuales generalmente son cubiertas por cada uno de los participantes); y promover alianzas entre las pymes (Rosales, 1997).

Con el acelerado proceso de la globalización, en la actualidad, es complicado para las pymes proceder 
de manera individual, dado que presentan deficiencias en cuanto al tamaño que hacen difícil la incorporación de tecnología y sistemas operativos, lo cual no permite la penetración de nuevos mercados, por lo cual no pueden obtener resultados positivos. Es por ello que es conveniente que las pymes se adapten a la realidad del mercado, es decir, es necesaria la interacción con los agentes cercanos que permiten la conformación de redes, las cuales afronten tanto las debilidades, como las amenazas y, especialmente, aprovechen todo tipo de oportunidad (Liendo, 2001).

Asimismo, Pallares (2004) menciona que la asociatividad empresarial es una forma de enfrentar los nuevos escenarios globales, los cuales traen consigo una liberación económica, desregulación y competencia. Además de esto, relaciona la asociatividad empresarial con la asociatividad territorial, esto es, la cooperación entre varios actores locales que buscan promover y alcanzar el desarrollo sostenible y, específicamente, crear una vinculación competitiva del territorio con el mundo. Por otro lado, se destaca que la asociatividad territorial esté basada en la coo-petencia, es decir, debe existir una cooperación constante entre los agentes que pertenecen al territorio, y así competir de manera directa y/o indirecta con los productos y empresas de otros territorios del país (Canzanelli y Pallares, 2011). Los modelos asociativos no son muy comunes, debido a que no existe un incentivo por parte del gobierno y hay una falta de cultura de cooperación existente en la mayoría de las pymes.

De acuerdo con lo anterior, se presentan las principales características de la asociatividad empresarial (Samaniego, 2009):

- Una incorporación de manera voluntaria, de mutuo acuerdo, en la cual bajo ninguna causa, las empresas están forzadas a integrar un grupo. Por lo contrario, actúan cuando tienen la plena convicción de que esto puede generar beneficios, crecimiento y oportunidades de desarrollo.

- Las empresas mantienen su autonomía, las decisiones de cada agente no deben estar en función de los demás. Es decir, cada agente decide qué hacer con los beneficios obtenidos.

- Principio de convivencia voluntaria e igualitaria con respecto al poder; todos los agentes pertenecientes a la asociatividad tienen el mismo poder, deber y derecho en la toma de decisiones.

- No hay existencia de subordinación, es decir, la identidad jurídica es independiente de los socios, se mantiene la cultura y estructura de gestión de los miembros y, por último, se excluyen fusiones, acuerdos, etc.

- No se excluye a ninguna empresa. La aplicación del fenómeno de asociatividad va dirigido a todo tipo de empresa, sin importar el tipo de industria al cual pertenece.

- Coordinación de acciones futuras y acuerdos explícitos, ya sean de tipo verbal o escrito, o transferencia permanente de recursos.

- Consecución de un objetivo común; con esto el acuerdo debe proporcionar beneficios a todos los participantes, generando un juego de suma positiva y los socios deben participar activamente para desarrollar sus habilidades.

\section{Ventajas, desventajas, estrategias y beneficios}

Con base en tabla 7, se encuentra que la asociatividad es una herramienta útil de cooperación que presenta más ventajas que desventajas, y permite un desarrollo económico y territorial en el que todos los agentes tienen un beneficio mutuo, con el fin de generar un aumento en la productividad y la competitividad. No obstante, las empresas deben tener una iniciativa propia que permita la implementación de la asociatividad como una alternativa de crecimiento. Con esto, las pymes serían un gran generador de empleo, un factor positivo de crecimiento en economías regionales en las que se incentivan y estimulan las capacidades individuales.

En la mayoría de los casos, los modelos de asociatividad permiten una disminución de los costos, acceso a tecnología y, especialmente, la transferencia de conocimiento. Esto último se comprende como uno de los beneficios más importantes que obtienen los agentes, ya que se está en un proceso constante de aprendizaje, en el cual existe un intercambio de experiencias que permiten no caer en el mismo error, o de reconocer oportunidades. El hecho de tener objetivos comunes claros a un corto, mediano y largo plazo, los cuales van guiados hacia el desarrollo sostenido y sustentable, permiten una mayor eficiencia en el funcionamiento de la asociatividad.

Se considera que otro de los grandes beneficios que se obtienen en los procesos de asociatividad es el aumento del poder de negociación con proveedores, productores, competidores y clientes, tanto a nivel nacional, como internacional, lo cual genera un mayor valor agregado, aumento de la calidad y cantidad de bienes y servicios producidos. Desde un principio, 
Tabla 7

Ventajas, desventajas y estrategias del VC

\begin{tabular}{|c|c|}
\hline Características & Descripción \\
\hline Ventajas & $\begin{array}{l}\text { - Aumento de la productividad } \\
\text { - Incremento poder de negociación } \\
\text { - Nuevos acceso a tecnología } \\
\text { - Transferencia de conocimientos } \\
\text { - Que las empresas compartan sus } \\
\text { recursos } \\
\text { - Minimización de riesgos } \\
\text { - Reducción de costos } \\
\text { - Se comparten riesgos y costos } \\
\text { - Mayor acceso a la financiación } \\
\text { - Mejora en la calidad y el diseño } \\
\text { - Aumento en la gestión del conoci- } \\
\text { - Miento técnico } \\
\text { - titividad y productividad } \\
\text { - Las empresas obtienen beneficios } \\
\text { de acuerdo a su grado de participa- } \\
\text { ción y compromiso } \\
\text { Efectos secundarios: una mentali- } \\
\text { dad más abierta, mayor confianza, } \\
\text { una visión a largo plazo }\end{array}$ \\
\hline Desventajas & $\begin{array}{l}\text { - Inconveniente con los agentes a la } \\
\text { hora de realizar una solicitud para } \\
\text { préstamos o créditos, ya que no } \\
\text { poseen una firma colateral } \\
\text { - No incentivos por parte del } \\
\text { gobierno } \\
\text { - No hay cultura de cooperación } \\
\text { - No existe en los participantes una } \\
\text { clara vocación de cooperación, } \\
\text { sino más bien, un alto grado de } \\
\text { desconfianza } \\
\text { - Por lo general no se definen reglas } \\
\text { del juego } \\
\text { - Los intereses de los agentes no } \\
\text { están alineados }\end{array}$ \\
\hline Estrategias & $\begin{array}{l}\text { - Estrategias colectivas } \\
\text { - Conformación de redes horizonta- } \\
\text { les de producción } \\
\text { - Benchmarking } \\
\text { - Cadena de valor como sistema } \\
\text { único } \\
\text { - Esquemas organizacionales }\end{array}$ \\
\hline
\end{tabular}

Nota. Elaboración propia a partir de Proyecto de cooperación, por C. D. Nicaragua, 2010.

se reconoció la importancia de la asociatividad en los territorios, ya que factores como la explotación de los recursos, el respeto y el cuidado por el medio ambiente permiten un desarrollo sostenible.

De igual manera, los agentes - bien sean personas o bien empresas - poseen una mejor disposición y capacidad de responder a las tendencias, cambios y señales del mercado, implementando productos innovadores, los cuales se diferencian a través de marcas, estilos, calidad y precios. Por otro lado, es importante mencionar que el aumento de la calidad de vida se evidencia en gran proporción, ya que tanto empleadores como empleados se benefician (Acevedo y Buitrago, 2009).

\section{Etapas del proceso asociativo}

Según Liendo (2001), el proceso de asociatividad empresarial tiene cinco fases que se clasifican de la siguiente forma:

1. Etapa de gestación: los agentes tienen interés en iniciar o crear un grupo. Aquí aparecen aspectos como la creación e integración de los agentes para un bien común, momento en que se analizan ventajas, desventajas y el potencial de cada una de las empresas que quieren ser partícipes de este proceso.

2. Etapa de estructuración: los empresarios tienen claro sus objetivos y aceptan las herramientas para alcanzar los objetivos planteados. Por lo general, en esta etapa, se definen las estrategias orientadas al cumplimiento de dichos objetivos.

3. Etapa de madurez: los agentes crean una identidad y propia y, por último, existe claridad y consistencia sobre las acciones a ejecutar. Se define la forma jurídica y las bases de auto-sostenibilidad del grupo.

4. Etapa productiva o de gestión: en esta etapa, se ejecutan todas las acciones pertinentes para obtener resultados positivos. De igual modo, se aceleran los procesos operativos internos que permitan obtener una gestión empresarial.

5. Etapa de declinación: en esta etapa, es común observar que los rendimientos empiezan a disminuir. La relación de las variables costo-beneficio del grupo son realmente negativas, es decir, los agentes deben tomar decisiones que determinen la desintegración parcial y/o total de la asociatividad, o crear nuevas estrategias.

\section{Principales tipos de asociatividad}

Los tipos de asociatividad se agrupan dependiendo de las necesidades de los agentes, es decir, se puede ubicar en la fase del proceso empresarial que desee: ya sea de diseño y comercialización, o que se relacione con cada una de las funciones básicas que existen en cualquier empresa. 
1. Red empresarial: se define como el conjunto de empresas que comparten información, procesos o simplemente conglomeran ofertas, sin dejar de funcionar de manera independiente. No hay una relación de subordinación y por lo general mantienen vínculos cooperativos para realizar acciones conjuntas. Por otra parte, Cardona (2000) explica las redes como principal componente de las cadenas productivas, es decir, como un conjunto de relaciones entre las unidades productivas para la superación de obstáculos y la conquista de nuevos mercados que sobrepasan los alcances individuales.

2. Cadena productiva: son sistemas que permiten la interacción de diferentes participantes de manera directa e indirecta en la producción de bienes o servicios, hasta el consumo de los mismos. Es decir, la cadena productiva muestra alternativas de crecimiento para entidades económicas de menor magnitud en procura de que aumenten los niveles de competitividad y la productividad (Tomta y Cbiatoua, 2009).

3. Clúster: se define como un tipo de asociación que se basa en la concentración geográfica y sectorial de diversas empresas, las cuales tienen una especialización, son capaces de crear procesos de innovación y conocimiento especializado, las cuales cooperan entre sí, pero trabajan a nivel individual en cuanto a la competencia (Lazzereti, 2004).

4. Distrito industrial: es un modelo de organización de la producción que tiene un enfoque de desarrollo económico y se define como entidades que se asocian como la comunidad y se relacionan mutuamente. Son generadores de innovación y difusión tecnológica (Becattini, 2004).

5. Alianza estratégica: según Acevedo y Buitrago (2009), "son acuerdos de organización y políticas de operación, donde empresas separadas comparten autoridad administrativa, establecen relaciones sociales y una posición conjunta. Existe una relación fundamental de cooperación de responsabilidades en un proyecto".

\section{Conclusiones}

- RSE es un concepto fundamental de gestión empresarial con carácter ético, social, político, cultural, ambiental y económico, el cual debe infundirse desde la academia.

- Los tres factores fundamentales de la RSE son: los agentes o los actores participes (más conocidos como grupos de interés o stakeholders); el medio ambiente (hace referencia al entorno en el que se desenvuelven dichos actores); y la empresa como motor de bienestar (la combinación de los dos primeros, como la empresa tras sus actuaciones, repercuten en el bienestar del ambiente).

- Existe una tipificación en cuanto a la RSE (tablas 1 y 2), a fin de establecer bajo qué normatividad una empresa debe actuar y ser socialmente responsable y cómo puede llegar a obtener algún tipo de certificación por ello, según le ajuste.

- Falta mayor precisión por parte de las políticas empresariales, en busca de fortalecer negocios de confianza y con mayor impacto social, los cuales evidencien los cambios territoriales en las comunidades.

- El concepto de RSE no es producto de la contemporaneidad, toma forma en la actualidad empresarial, en busca de asentar percepciones empresariales justas y equitativas.

- Porter y Kramer (2011), en un artículo de la revista Harvard Business Review, hacen eco de las reflexiones de sus colegas y denuncian lo que denominaban "El juego de los ratings". En el artículo, Porter y su colega criticaban los ratings existentes, acusándolos de falta de consistencia en sus mediciones y de no reflejar de manera correcta el impacto social de las compañías:

Los criterios usados en los diferentes ratings varían enormemente. El Dow Jones Sustainability Index, por ejemplo, incluye en sus evaluaciones aspectos como el desempeño económico y concede al servicio al cliente un peso casi un $50 \%$ más alto que a las actividades relacionadas con la ciudadanía corporativa (Transparencia, 2007).

- La RSE es más que un certificado para mostrar, es un impacto social que debe permear los actores internos y externos. Por ello, el concepto ha ido ampliándose e incluyendo teorías que lo acentúan como el comercio justo, negocios inclusivos y valor compartido.

- El compromiso de los nuevos empresarios o empresarios renovados se debe pensar en el progreso de la calidad de vida de sus empleados y el buen vivir de sus familias, logística inversa, sostenibilidad de la cadena de suministro, en la comunidad que rodea su organización, cumplir con los compromisos que tiene con el gobierno, ser transparente con la información que suministra a sus 
inversionistas, respetar profundamente el medio ambiente que usa para sus procesos productivos o de servicios, y entregar a sus clientes productos sanos con precios justos.

- La debe y el valor compartido no es un tema que pertenezca solamente a la gran empresa, es un compromiso del comercio y la industrialización en general.

- Las mipymes deben concebir en sus estrategias empresariales los conceptos profundizados anteriormente, ya que les va a permitir ser reconocidas con mayor rapidez entre los mercados nacionales y globales.

- Los conceptos de negocios inclusivos y valor compartido deben hacer parte de las políticas empresariales, y concebirse como un tema de humanización empresarial.

- Estos temas deben ser transversales a las políticas institucionales empresariales y desarrollarse uno a uno, según la capacidad económica y el compromiso social de cada empresa, en busca de crear negocios asociativos.

- Hacer asociatividad demuestra el compromiso de crecimiento económico compartido.

Esfuerzos cooperados coadyuvan a las empresas a ser más sostenibles y perdurables en el tiempo.

\section{Referencias}

AccountAbility Organization (2012). Estándares. Recuperado de http:// www.accountability.org/standards/

Acevedo, M. y Buitrago, M. (2009). Asociatividad empresarial, crecimiento productivo e innovación. el caso de las PYMES del sector textil y confecciones en Bogotá. Bogotá: Universidad de la Salle.

Barcenas, J. (2015). Y mi empresa... ¿hace algo por el valor compartido? Dinero, Disponible en http://www. dinero.com/opinion/columnistas/articulo/opinion-acerca-importancia-del-valor-compartido-empresas/214871

Barrat Brown, M. (1998). Comercio justo, comercio injusto: hacia una cooperación internacional. Barcelona: Icara.

Becattini. (1979). Scienze economica e trasformazioni socile. La Nuova Italia, 9-32.

Becattini, G. (2004). Vicisitudes y potencialidades de un concepto: el distrito industrial. Economía Industrial, 359 .

Bovard, J. (1991). The fair trade fraud. New York: Palgrave Macmillan.
Bowen, H. (1953). Social responsibilities of the businessman. New York: Harper \& Row.

Canzanelli, G. y Pallares, Z. (2011). Cartilla-guía del desarrollo económico local en Colombia. Bogotá: RED ADELCO.

Cardona, M. (2000). Redes sociales en la cadena productiva de la industria del vestido. Los casos de Monterrey (México) y Medellín (Colombia). Medellín: Colecciones Fondo Editorial EAFIT.

Carroll, A. B. (1991). The pyramid of corporate social responsibility: toward the moral management of organizational stakeholders. Business Horizons, 34(4), 39-48.

Cecodes. (2008). Los negocios inclusivos. Recuperado de http:// www.snvworld.org/files/publications/los_negocios_inclusivos_en_colombia.pdf

Comisión Europea. (10 de Marzo del 2001). Libro verde: fomentar un marco europeo para la responsabilidad social de las empresas. Recuperado de http://eur-lex.europa. eu/LexUriServ/LexUriServ.do?uri=COM:2001:0366:FIN:es:PDF

De la Torre, F. J. (2004). Comercio justo: una cuestión de ética. En SETEM, El comercio justo en España 2004 (pp. 9-18). España: Icaria.

Duque, Y., Cardona, M., y Rendón, J. (2013). Responsabilidad social empresarial: teorías, índices, estándares y certificaciones. Cuadernos de Administración, 196206.

Drucker, P. (1984). The new meaning of corporate social responsibility. California Management Review, 26(2), 53-63.

Ransom, D. (2011). The No-Nonsense Guide to Fair Trade. New Internationalist blog. Recuperado de http://newint.org/blog/books/2011/02/07/weekly-no-nonsenseguide-fair-trade/\#sthash.rXXYIlbo.dpuf

Drucker, P. (1996). Desafíos de la administración el siglo XXI. Mc Graw Hill.

Frederick, W. (1960). The growing concern over business responsibility. California Management Review, 2(4), 54-61.

Friedman, M. (1970, septiembre 13). The social responsibility of business is to increase its profits. The New York Times Magazine, 1-6.

Fumio, S. Discurso de apertura. (xxI Congreso Mundial del Apostolatus Maris). Río de Janeiro, Octubre 5 del 2002.

Global Reporting Initiative Organization. (2012). About GRI. Recuperado de https://www.globalreporting.org/ information/about-gri/Pages/default.aspx

Heald, M. (1957). Management's Responsibility to Society: 
The Growth of an Idea. Business History Review, 31(4): 375-384.

International Organization for Standardization. [ISO]. (2010). Iso. Recuperado de http://www.iso.org/iso/ iso_26000_project_overview-es.pdf

Joon Chang, H. (2004). Retirar la escalera: la estrategia del desarrollo en perspectiva histórica. Madrid: La Catarata.

Lazzereti, L. (2004). Distritos industriales cluster y otros: un análisis entre la economía industrial y la gestión estratégica. España: Economía Industrial.

Liendo, M. (2001). Asociatividad una alternativa para el desarrollo y crecimiento de las PyMEs. Sextas Jornadas "Investigaciones en la facultad" de Ciencias Económicas y Estadística, 300-319. Argentina: Universidad Nacional de Rosario.

Ligteringen, E. (2005). Future of corporate responsability codes, standards and frameworks. Recuperado de www. accountability21.net/uploadstore/cms/docs/ Landscape\%Paper.pdf

McGuire, J. (1963). Business and society. New York: McGraw-Hill.

Pesce, D. (2006). La RSE en el contexto internacional y la norma Iso 26000 de Responsabilidad Social. Chile: Pontificia Universidad Católica de Valparaiso.

Porter, M. y Kramer, M. (2006, diciembre). Estrategia y Sociedad. Harvard Business Review: América Latina, $1-15$.

Porter, M. y Kramer, M. (2011, enero). La creación de valor compartido. Harvard Business Review: América Latina, 1-18. Recuperado de http://peru2021.org. pe/C601D503-E796-42D5-8083-733F7D6413F3/FinalDownload/DownloadId-5F794924F35933B19C599F5CF72E935B/C601D503-E796-42D5-8083-733F7D6413F3/ repositorioaps/0/0/par/creacionvalorcompartido/shared\%20value\%20in\%20spanish.pdf

Red Empresarial de Negocios Inclusivos. (s. f.) Información. Red Empresarial de Negocios Inclusivos. Presentación PowerPoint.

Transparencia, F. C. (2007). Rankings, ratings, índices y demás libros de caballería. Recuperado de http://www. compromisoempresarial.com/opinion-rsc-ong-management/2007/08/rankings-ratings-indices-y-demas-libros-de-caballeria/
Nicaragua, C. D. (2010). Proyecto de cooperación. Managua. Organización de las Naciones Unidas para la Alimentación y la Agricultura. [FAo]. (2007). Documentos archivo. Recuperado de http://www.fao.org/docrep/ 007/ad818s/ ad818s06.htm

Pacto Mundial. (2013). Quiénes somos. Recuperado de http:// www.pactomundial.org/global-compact/

Pallares, Z. (2004). Asociatividad empresarial estrategia para la competitividad. Bogotá DC: Fondo Editorial Nueva Empresa.

Rosales, R. (1997). La asociatividad como estrategias de fortalecimiento de las Pymes. Cambios estratégicos en las políticas industriales, 51. SELA.

Sichar G. (2002). Comercio justo ¿una alternativa real? Madrid: Fundación CIDEAL y SETEM.

Smith, A. (2004). Teoría de los sentimientos morales. México: Fondo de Cultura Económica.

Strandberg, L. (2010). La medición y la comunicación de la RSE: indicadores y normas. Cuadernos de la Cátedra "La Caixa" de Responsabilidad Social de la Empresa y Gobierno Corporativo, 9. Recuperado de http:// www.iese.edu/es/ad/ catedras/caixa/Newsletters2009/ Newsletter9FormatoBase.asp

SNV/WBCSD. (2008, enero). Negocios inclusivos. Iniciativas empresariales rentables con impacto en el desarrollo. Recuperado de http://www.cecodes.org.co/ 28983ACFAEA0-4356-9435-402BCFC7F717/FinalDownload/ DownloadId-60953CC7AFECAF6BF4F34E19E2CF9 993/28983ACF-AEA0-4356-9435-402BCFC7F717/ descargas/documentos_ni/brochure_alianza_wbcsd_ snv.pdf

Tomta, D. y Cbiatoua, C. (2009). Cadenas productivas y productividad de las Mipymes. Criterio Libre, 11(7), 145-164.

Vivas, E. y Montagut, X. (2006). ¿Adónde va el comercio justo? Barcelona: Icara.

Zadek, S. (agosto del 2005). El camino hacia la responsabilidad corporativa. Harvard Business Review, Edición especial: el factor social, 54-63.

Zambrano, P. (2011). Ha Joon Chang (1963). Blog de Pablo Zambrano Pontón. Recuperado de http:// pablozambranoponton.blogspot.com.co/2011/03/hajoon-chang.html 